EPSC Abstracts

Vol. 14, EPSC2020-190, 2020, updated on 04 Dec 2021

https://doi.org/10.5194/epsc2020-190

Europlanet Science Congress 2020

(C) Author(s) 2021. This work is distributed under

the Creative Commons Attribution 4.0 License.

\title{
Virtual European Solar \& Planetary Access (VESPA): Progress and prospects
}

Stéphane Erard ${ }^{1}$, Baptiste Cecconi ${ }^{1}$, Pierre Le Sidaner ${ }^{2}$, Angelo Pio Rossi ${ }^{3}$, Carlos Brandt ${ }^{3}$, Hanna Rothkaehl ${ }^{4}$, Lucasz Tomasik ${ }^{4}$, Stavro Ivanovski ${ }^{5}$, Marco Molinaro ${ }^{5}$, Bernard Schmitt ${ }^{6}$, Vincent Génot ${ }^{7}$, Nicolas André ${ }^{7}$, Ann Carine Vandaele ${ }^{8}$, Loic Trompet $^{8}$, Manuel Scherf ${ }^{9}$, Ricardo Hueso ${ }^{10}$, Anni Määttänen ${ }^{11}$, Ehouarn Millour ${ }^{12}$, Frédéric Schmidt ${ }^{13}$, Ingo Waldmann ${ }^{14}$, and the VESPA team ${ }^{*}$ ${ }^{1}$ LESIA / Observatoire de Paris, LESIA, Meudon, France (stephane.erard@obspm.fr)

${ }^{2}$ DIO-VO, Obs. Paris/CNRS, Fr

${ }^{3}$ Jacobs University, Bremen, Germany

${ }^{4}$ CBK-PAN, Warsaw, Poland

${ }^{5}$ INAF/OATS, Trieste, Italy

${ }^{6}$ IPAG UGA/CNRS, Grenoble, Fr

${ }^{7}$ IRAP/CNRS/UPS/CNES, Toulouse, Fr

${ }^{8}$ BIRA/IASB, Brussels, Be

${ }^{9} \mathrm{OeAW}, \mathrm{Graz}$, Aust

${ }^{10}$ UPV/EHU, Bilbao, Spain

${ }^{11}$ LATMOS/IPSL, Sorbonne Université, UVSQ, U. Paris-Saclay, CNRS, Guyancourt, Fr

${ }^{12}$ LMD/IPSL/CNRS, Paris, Fr

${ }^{13}$ GEOPS/IPSL/CNRS/UPS, Orsay, Fr

${ }^{14}$ University College London, UK

${ }^{*}$ A full list of authors appears at the end of the abstract

The H2020 Europlanet-2020 programme, which ended on Aug $31^{\text {st }}, 2019$, included an activity called VESPA (Virtual European Solar and Planetary Access), which focused on adapting Virtual Observatory (VO) techniques to handle Planetary Science data [1] [2]. The outcome of this activity is a contributive data distribution system where data services are located and maintained in research institutes, declared in a registry, and accessed by several clients based on a specific access protocol. During Europlanet-2020, 52 data services were installed, including the complete ESA Planetary Science Archive, and the outcome of several EU funded projects. Data are described using the EPN-TAP protocol, which parameters describe acquisition and observing conditions as well as data characteristics (physical quantity, data type, etc). A main search portal has been developed to optimize the user experience, which queries all services together. Compliance with Vo standards ensures that existing tools can be used as well, either to access or visualize the data. In addition, a bridge linking the VO and Geographic Information Systems (GIS) has been installed to address formats and tools used to study planetary surfaces; several large data infrastructures were also installed or upgraded (SSHADE for lab spectroscopy, PVOL for amateurs images, AMDA for plasmarelated data).

In the framework of the starting Europlanet-2024 programme, the VESPA activity will complete this system even further: 30-50 new data services will be installed, focusing on derived data, and 
experimental data produced in other Work Packages of Europlanet-2024; connections between PDS4 and EPN-TAP dictionaries will make PDS metadata searchable from the VESPA portal and vice versa; Solar System data present in astronomical VO catalogues will be made accessible, e.g. from the VizieR database. The search system will be connected with more powerful display and analysing tools: a run-on-demand platform will be installed, as well as Machine Learning capacities to process the available content. Finally, long-term sustainability will be improved by setting VESPA hubs to assist data providers in maintaining their services, and by using the new EU-funded European Open Science Cloud (EOSC). In addition to favoring data exploitation, VESPA will provide a handy and economical solution to Open Science challenges in the field.

The Europlanet 2020 \& 2024 Research Infrastructure project have received funding from the European Union's Horizon 2020 research and innovation programme under grant agreements No $654208 \& 871149$.

[1] Erard et al 2018, Planet. Space Sci. 150, 65-85. 10.1016/j.pss.2017.05.013. ArXiv 1705.09727

[2] Erard et al. 2020, Data Science Journal 19, 22. doi: 10.5334/dsj-2020-022.

VESPA team: S. Erard (1), B. Cecconi (1), P. Le Sidaner (2), A. P. Rossi (3), C. Brandt (3), H. Rothkaehl (4), L. Tomasik, (4), S. Ivanovski (5), M. Molinaro (5), B. Schmitt (6), P. Bollard (6), D. Albert (6), V. Génot (7), N. André (7), A. C. Vandaele (8), L. Trompet (8), M. Scherf (9), R. Hueso (10), A. Määttänen (11), E. Millour (12), F. Schmidt, (13), I. Waldmann (14), P. Fernique (15), M. D'Amore (16), M. Demleitner (17), N. Manaud (18), M. Taylor (19), I. Alexeev (20) 